\title{
método de mando automáticoo de una fábrica de cemento mediante un computador
}

Preparación de crudos, horno y enfriador

J. HAYES, Ingeniero E. P. IBM Francia

F. RAIS, Ingeniero E. P. F. IBM Suiza

\section{INTRODUCCION}

Las aplicaciones potenciales de un computador en una fábrica de cemento moderna son múltiples:

- vigilancia del conjunto de la fábrica;

- gestión racional de cantera;

- elaboración de la mezcla óptima de crudos;

- mando automático de los talleres:

- de preparación de la materia prima,

- de cocción (horno y enfriador),

- de molienda del clínker;

- gestión de los depósitos de piezas de recambio y establecimiento de calendarios de conservación;

- elaboración de informes de funcionamiento que proporcionan a la dirección las cifras con las que puede evaluar la marcha de la instalación, taller por taller.

Esta comunicación tiene por objeto presentar:

- la resolución de un problema de mezcla de crudos con un computador conectado directamente a los dosificadores;

- los métodos de mando automático de un conjunto horno-enfriador en vía seca. 


\section{MEZCLA DE CRUDOS}

\section{Condiciones de automatización}

El tratamiento en computador del problema de mezcla de crudos depende en primer lugar de la calidad de las materias primas. Los componentes que definen la mezcla son esencialmente: $\mathrm{CaO}, \mathrm{SiO}_{2}, \mathrm{Al}_{2} \mathrm{O}_{3}, \mathrm{Fe}_{2} \mathrm{O}_{3}$ y $\mathrm{MgO}$. Su distribución puede ser muy diferente de una cantera a otra; en ciertos casos, las cantidades de componentes secundarios permanecen dentro de los límites compatibles con una cocción correcta: en este caso pueden despreciarse en la definición de la mezcla. En otros casos, hay que tener en cuenta todos los componentes para obtener una mezcla utilizable. Las variaciones en el tiempo de las composiciones de cada silo de alimentación desempeñan un papel determinante: ciertas canteras proporcionan un material de tal constancia que el control de la mezcla no ofrece dificultades. Otras instalaciones presentan variaciones tan rápidas que un muestreo efectuado cada 20 minutos no es suficiente para representar correctamente las composiciones.

\section{Objetivos de la automatización}

El usuario del computador debe fijar estos objetivos. Puede limitarse a fijar un valor de consigna para dos o tres componentes; puede también imponer los valores de los módulos que determinan la calidad de la mezcla. En el caso de que se disponga de un gran número de materias primas, es ventajoso optimizar el precio de coste ciñéndose lo más posible a los objetivos técnicos. Por lo que respecta al programa de regulación numérica, estos objetivos se traducen por valores de consigna, fijos o variables en el tiempo.

\section{Ejemplo de aplicación (fig. 1)}

Tal aplicación se ha desarrollado ya con éxito en un ordenador IBM 1800. El análisis de la materia se realiza a la salida de un molino de bolas mediante un analizador de rayos $\mathrm{X}$, que trabaja de modo continuo en cuatro canales $(\mathrm{Ca}, \mathrm{Al}, \mathrm{Si}, \mathrm{Fe})$. Tres silos alimentan el molino de martillos: los silos de caliza, de arcilla y de pirita. El grado de llenado del molino está regulado por la cantidad total de materia introducida. Las medidas empleadas son:

- un nivel de ruido;

- las temperaturas en los separadores;

- las potencias de los molinos de martillos.

En el sistema quedan dos grados de libertad utilizados para mantener constantes las composiciones de $\mathrm{CaO}$ y $\mathrm{Fe}_{2} \mathrm{O}_{3}$. El metodo puesto en marcha es el siguiente: para cada silo que hay que llenar a la salida dol molino, se dan los valores de consigna do $\mathrm{CaO}$ y $\mathrm{Fe}_{2} \mathrm{O}_{3}$. 
Durante el llenado, se determina, mediante la balanza de salida y los análisis, el grado de llenado y la composición del silo; de aquí se deducen nuevos valores de consigna para $\mathrm{CaO}$ y $\mathrm{Fe}_{2} \mathrm{O}_{3}$ que corrigen la composición con las $\mathrm{N}$ toneladas siguientes. Hay que señalar que la materia analizada en cada momento a la salida del molino se ha introducido aproximadamente veinte minutos antes: el paso del crudo a través del molino lo simula el ordenador, que predice las variaciones de composición a la salida en un intervalo de 20 minutos. El resultado de esta predicción se utiliza para controlar las variaciones en los dosificadores. Además, la composición de cada uno de los silos de alimentación se determina y corrige continuamente: el ordenador analiza las variaciones medidas de composición a continuación de los controles aplicados en los dosificadores, teniendo en cuenta la corrección del tiempo de paso del material.

\section{Resultados obtenidos}

La composición del crudo que sale de la planta de molienda es más constante que con los controles clásicos; se puede reducir considerablemente el tiempo de homogeneización con los silos llenos.

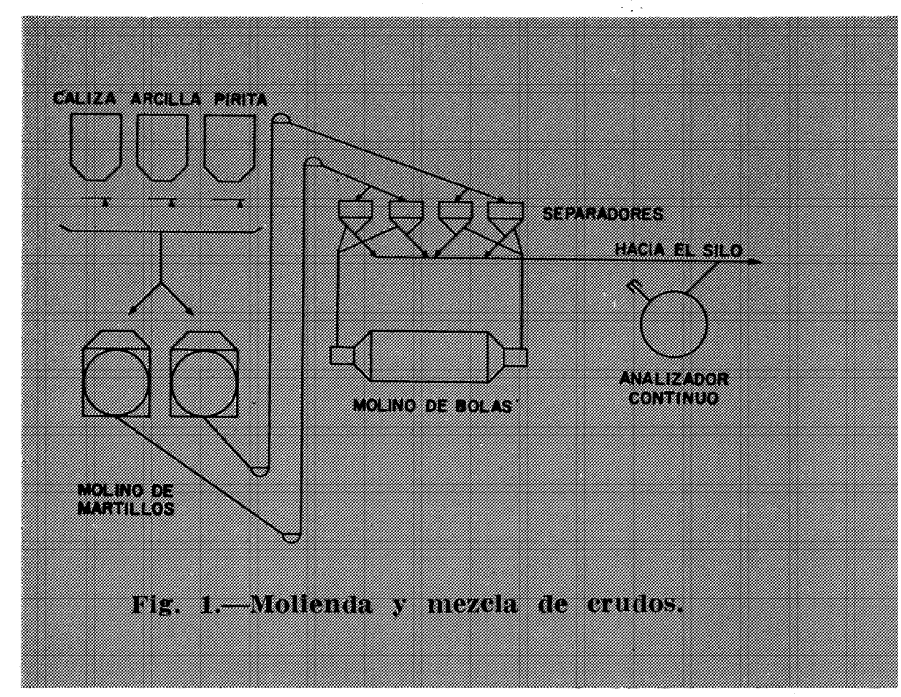

El contenido de cada silo corresponde al valor prescrito, lo que hace innecesarias las correcciones de silos que exigían antes los análisis efectuados después de la homogeneización, no exigiendo por tanto la segunda homogeneización.

La cocción se beneficia igualmente de la constancia de composición del crudo.

\section{MANEJO DE UN CONJUNTO HORNO-ENFRIADOR}

\section{Objetivos}

Los objetivos fijados al partir de un estudio-tipo son los siguientes:

- marcha del horno:

- mantener la temperatura en la zona de cocción dentro de unos valores dados,

- asegurar un porcentaje de oxígeno en los humos;

- marcha del enfriador:

- asegurar un enfriamiento correcto del clínker,

- proteger la parrilla en caso de avalanchas de material,

- mantener la temperatura del aire secundario en un valor lo más constante posible,

- mantener la presión en el cabezal del horno en un valor constante. 
El logro de estos objetivos ha dado ya lugar a diversos esquemas de regulación, que dependen particularmente de los métodos de análisis del problema, de las circunstancias técnicas impuestas y del material de control utilizado. Cuando este material de control comprende un ordenador, es importante aprovechar al máximo sus capacidades de intervención para reducir el consumo específico, alargar la vida del revestimiento refractario del horno y regularizar las condiciones de marcha.

\section{Marcha del horno (fig. 2)}

Las variables de mando utilizadas son:

- el caudal de combustible en el quemador $(F)$;

- la velocidad de rotación del horno (VN);

- la velocidad del ventilador de tiro (V 1).

La regulación del porcentaje de oxígeno por el tiro se efectúa por un bucle de regulación numérica independiente. El manejo del horno radica en la utilización de un modelo matemático con el que se puede calcular una temperatura de cocción extrapolada $\mathrm{TZ}[(\mathrm{k}+1) \Delta]$ a partir de los valores anteriores $\mathrm{TZ}[\mathrm{k} \Delta]$, $\mathrm{TZ}[(\mathrm{k}-1) \Delta]$, etc. .., y de los valores actuales y anteriores de los de control $\mathrm{F}$ y VN, así como de las perturbaciones $\mathrm{O}_{2}$ y $\mathrm{T}_{1}$. El modelo se traduce por una relación de recurrencia:

$$
\begin{aligned}
& \mathrm{TZ}_{\mathrm{k}+1}=\mathrm{f}\left[\mathrm{TZ}_{\mathrm{k}}, \quad \mathrm{TZ}_{\mathrm{k}-1}, \quad, \ldots \ldots \ldots \ldots \ldots\right. \\
& \mathrm{F}_{\mathrm{k}}, \quad \mathrm{F}_{\mathrm{k}-1}, \quad, \ldots \ldots \ldots \ldots \ldots \\
& \mathrm{VN}_{\mathrm{k}}, \mathrm{VN}_{\mathrm{k}-1},, \ldots \ldots \ldots \ldots \ldots \\
& \mathrm{O} 2_{\mathrm{k}}, \quad \mathrm{O} 2_{\mathrm{k}-1}, \quad, \ldots \ldots \ldots \ldots \ldots \\
& \left.\mathrm{T} 1_{\mathrm{k}}, \quad \mathrm{T} 1_{\mathrm{k}-1}, \quad, \ldots \ldots \ldots \ldots \ldots . . . . .\right]
\end{aligned}
$$

Señalemos que el modelo puede incluir explícitamente las variaciones del caudal de material si éste está sometido a frecuentes cambios. El cálculo de órdenes se efectúa cada 3 minutos (lo que corresponde, aproximadamente, al $1 / 10$ de la constante de tiempo dominante del proceso), a raíz de la predicción de TZ en un intervalo de una media hora. La lógica de marcha es tal que el algoritmo degenera en marcha por reacción pura cuando una gran perturbación viene a hacer poco precisos los valores previstos en un intervalo remoto.

El modelo quedó establecido a partir de campañas de medidas efectuadas sobre un horno de vía seca; se trata de un modelo dinámico lineal, en el cual la determinación de coeficiente se hace por un método de míni-

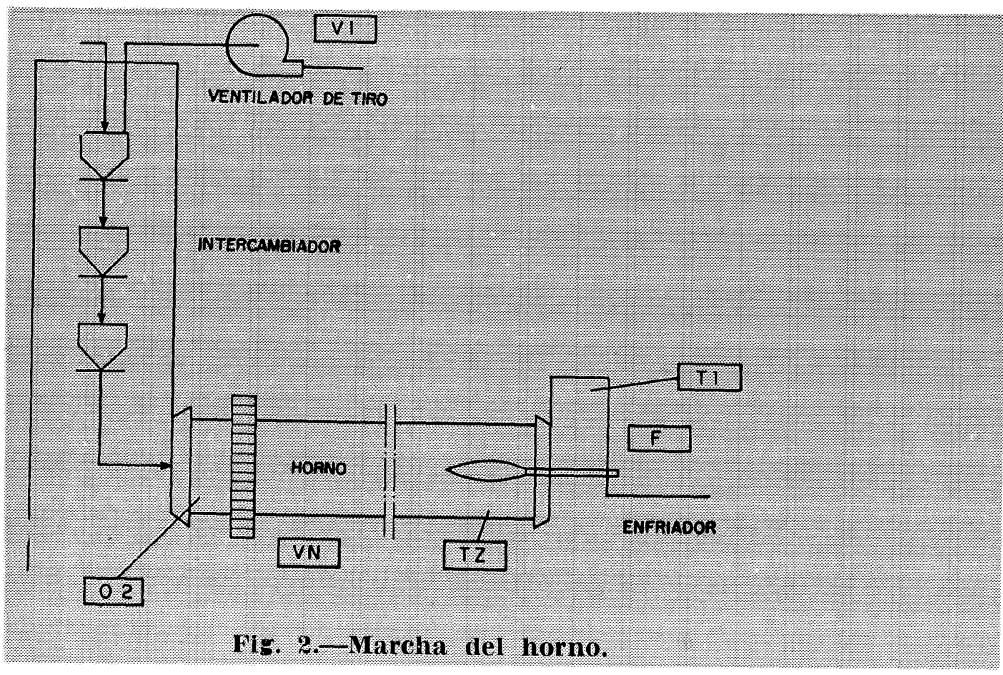
mos cuadrados sobre el historial de 8 horas aproximadamente. La validez del modelo se ensaya por simulación y comparación del resultado de las simulaciones con las medidas 
reales. Precisemos que, como en la marcha del enfriador, es indispensable filtrar las medidas con fórmulas apropiadas antes de utilizarlas, ya sea en marcha automática, o bien para determinar los coeficientes del modelo. En la figura 4 se indican resultados de simulación obtenidos con este método.

Además, se ha simulado también el conjunto horno-ordenador, que nos lleva a los resultados de la figura 4 . Se ve que el programa de marcha exige órdenes sobre el combustible más frecuentes y menos enérgicas que en la marcha manual, lo que se traduce en ahorro de combustible y en aumento de la vida del refractario.

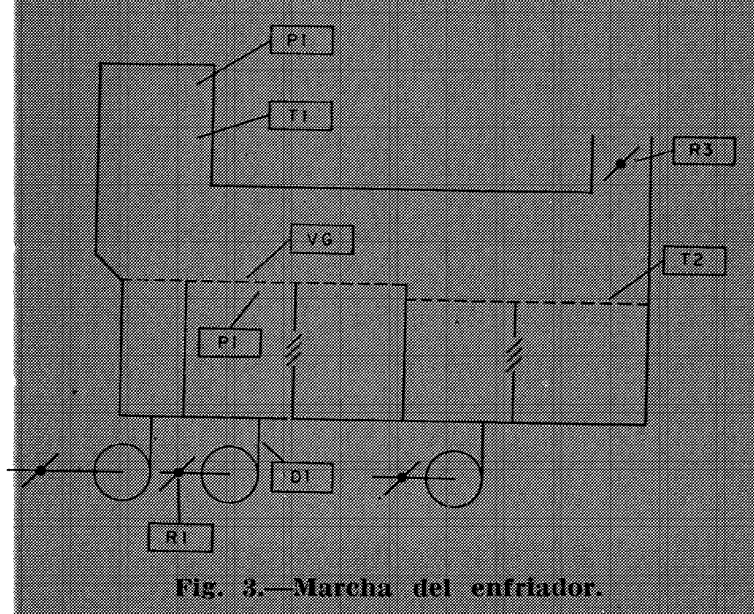

\section{Marcha del enfriador (fig. 3)}

Las variables de mando utilizadas en el enfriador estudiado son las siguientes:

- velocidad de parrilla (VG);

- registros de regulación de los caudales en los ventiladores soplantes $(\mathrm{R} 1, \mathrm{R} 2)$;

- registro de la chimenea de salida (R3).

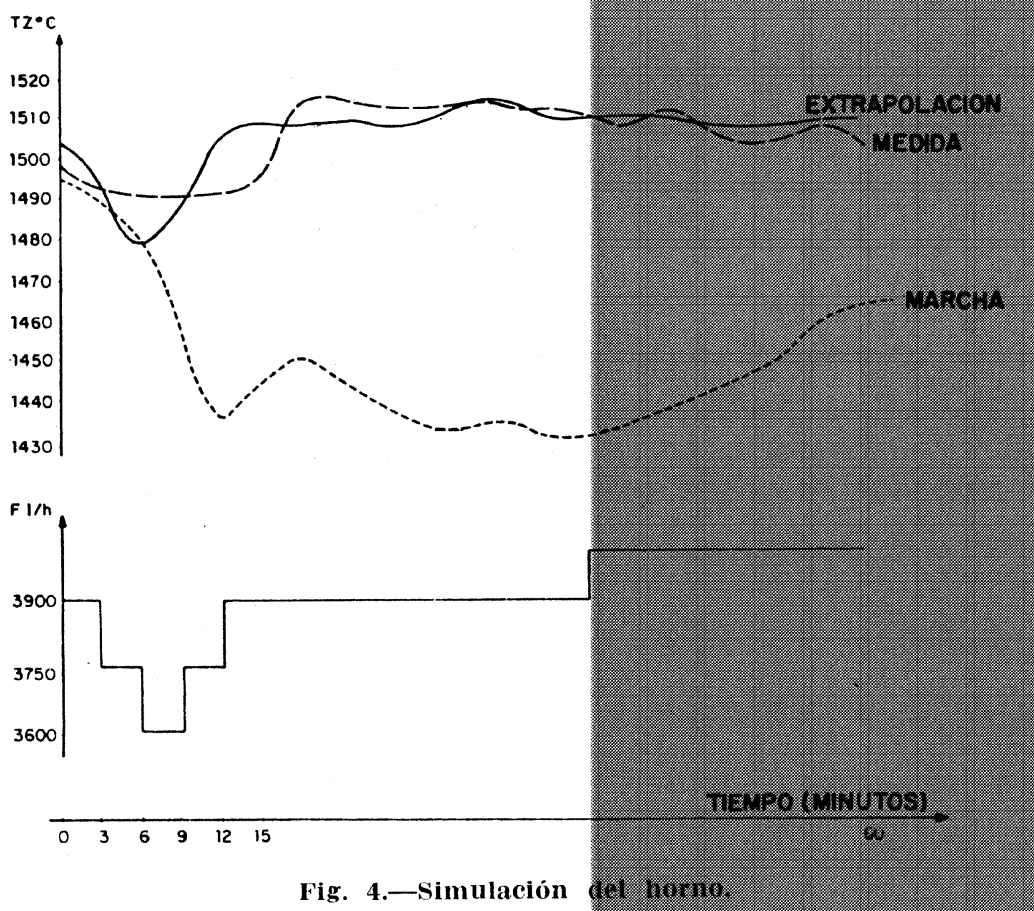

El mantenimiento de la presión P1 manejando el registro R3 es una regulación clásica sobre la que es inútil extenderse. Indiquemos, sin embargo, que el hecho de que 
se efectúe en forma numérica permite prever, en el caso de que R3 llegue a abrirse al máximo, una modificación prioritaria de caudal del ventilador inferior (más próximo a la chimenea).

El mantenimiento de la temperatura de aire secundario en un valor constante no puede, en la instalación estudiada, realizarse por una regulación directa basada en la medida de T1. Esta es en efecto delicada, ya que el captador se encuentra en contacto con gases a temperatura elevada cargados de polvo y circulando con una velocidad importante. Parece deseable lograr una constancia aproximada de T1 como resultado de otras dos regulaciones:

- regulación de la porosidad aparente de la capa de clínker por encima de los compartimientos superiores;

- regulación del caudal D1 del ventilador superior (más próximo al cabezal del horno).

Estas dos regulaciones presentan además la ventaja de asegurar la protección de la parrilla en el caso de que llegue una fuerte avalancha de material.

La porosidad aparente de la capa de clínker se valora a partir de la presión bajo la parrilla P1 y del caudal D1, y calculándose permanentemente. La variable de mando asociada es la velocidad de la parrilla; el algoritmo de regulación que hay que utilizar es un $\mathrm{P} 1$ clásico, traducido en números de la siguiente forma:

donde:

$$
\mathrm{VG}_{\mathrm{k}}=\mathrm{K} 1\left(\mathrm{e}_{\mathrm{k}}+\frac{\mathrm{T}}{\mathrm{T} 1} \sum_{\mathrm{k}} \mathrm{e}_{\mathrm{k}}\right)
$$

$\mathrm{VG}_{\mathrm{k}}=$ velocidad de parrilla a ordenar en el instante $\mathrm{kT}$.

$\mathbf{e}_{\mathrm{k}}=$ diferencia entre la porosidad en el instante $\mathrm{kT}$ y su valor consigna.

$\mathrm{K} 1, \mathrm{~T} 1=$ coeficientes de regulación

$\mathrm{T}=$ período de acción del programa de regulación.

La regulación del caudal D1 se efectúa por acción en el registro $R 1$. Se trata, de hecho, de un ajuste de estados estáticos, ya que la respuesta del caudal a una variación de posición de $\mathrm{R} 1$ es muy rápida, y que la precisión requerida no justifica un mando que tenga en cuenta la dinámica de este proceso. En cambio, tal modo se adapta bien a las variaciones de la parrilla que corresponden a frecuencias más bajas. El algoritmo utilizado es del tipo:

donde:

$$
\Delta \mathrm{R} 1=\mathrm{a}_{1} \Delta \mathrm{D} 1+\mathrm{b}_{1} \Delta \mathrm{P} 1,
$$

$\Delta \mathrm{R} 1=$ variación de posición del registro

$\Delta \mathrm{D} 1=$ error de caudal.

$\Delta \mathrm{P} 1=$ variación de presión.

$a_{1}$ es una constante.

$\mathrm{b}_{1}$ es, o bien nula, o bien igual a una constante según que la variación de presión P1 se deba a la abertura de R1 o a una perturbación del lecho de clínker. 
Este razonamiento se destina a aumentar la eficacia de la regulación en el caso de que se produzca una avalancha o una falta de material.

\section{SISTEMA DE MANDO}

\section{Descripción}

Tal sistema de mando automático comprende:

- captadores de medidas que dan señales eléctricas a la unidad de entrada del ordenador;

- el ordenador propiamente dicho;

- órganos reguladores que permitan actuar sobre válvulas, motores de velocidad variable, registros, etc....

El ordenador utilizado es el ordenador industrial IBM 1800; el sistema comprende esencialmente :

- una unidad aritmética y lógica, en cuya memoria central se almacenan una parte del programa de explotación TSX, proporcionado con el ordenador, así como el programa de aplicación que se está desarrollando;

- una unidad de memoria externa, compuesta por discos magnéticos, que recibe el conjunto de programas llamados sucesivamente por la unidad central, los ficheros de medidas, los resultados de los balances técnicos, etc....;

- una unidad de entrada-salida, en la que concurre el conjunto de señales de medida procedentes de los transmisores, las señales que indican el estado lógico de la instalación (estado de los conmutadores "manual-automático"), y los impulsos dados por los contadores de caudal de combustible. De esta unidad parten señales de órdenes a los diferentes órganos reguladores, en forma de impulsos, así como las señales continuas que dirigen los aparatos registradores;

- dos impresoras, con teclado alfa-numérico, que permiten dialogar con el ordenador: una se encuentra en la sala de control y la otra en la sala del ordenador;

- un lector-perforador de tarjetas, utilizado para la puesta a punto de nuevos programas y para la explotación del ordenador de marcha automático como un ordenador científico: el desarrollo de programas de comprobación de medidas y de cálculos diversos se inicia a partir del lector de tarjetas. La estación de "perforación" se utiliza, por ejemplo, para inscribir en las tarjetas los resultados de una campaña de medidas, con objeto de efectuar eventualmente su examen detenido en ordenadores más potentes.

\section{SISTEMA DE EXPLOTACION}

Este programa, que acompaña al ordenador, desempeña un papel esencial en la forma en que se maneja el conjunto. Tiene como fines esenciales:

- utilizar los tiempos libres del ordenador, para desarrollar los programas llamados "no-proceso" durante el mando automático de la instalación. Estos programas 
"no proceso" son, por ejemplo, los de impresión de informes de marcha, de escrutinio de medidas, etc...;

- permitir la escritura de programas de regulación y de mando automático en lenguaje FORTRAN: esto facilita, en pocos minutos, pasar de la concepción de un algoritmo de regulación, a su ensayo efectivo en la instalación;

- liberar al usuario de numerosas sujeciones debidas al aspecto "tiempo real" de la aplicación: gestión de sucesiones de espera de programas con prioridad entre sí y tratamiento inmediato de las "interrupciones", causadas por un incidente, que interrumpen el trabajo en marcha en el ordenador para tratar un programa más prioritario.

\section{v. CONCLUSION}

No hay duda de que la presencia de un ordenador en una fábrica es un factor de progreso considerable. Las facultades de memoria y de cálculo que se ponen a la disposición del ingeniero del cemento le permiten profundizar en los conocimientos que puede tener sobre su proceso. Además, la facilidad con la que es posible ensayar métodos nuevos de mando automático hace que estos métodos estén en continua evolución. Sin embargo, conviene señalar que el tiempo es un factor muy importante: cualquier modificación en los métodos de marcha automática supone una valoración de los beneficios obtenidos; esta valoración no puede hacerse seriamente sino tras el análisis de varios meses de marcha continua. 